\title{
PERIODS OF CONTINUOUS MAPS ON SOME COMPACT SPACES
}

\author{
JUAN LUIS GARCÍA GUIRAO ${ }^{1}$ AND JAUME LLIBRE ${ }^{2}$
}

\begin{abstract}
The objective of this paper is to provide information on the set of periodic points of a continuous self-map defined in the following compact spaces: $\mathbb{S}^{n}$ (the $n$-dimensional sphere), $\mathbb{S}^{n} \times \mathbb{S}^{m}$ (the product space of the $n$-dimensional with the $m$-dimensional spheres), $\mathbb{C} P^{n}$ (the $n$-dimensional complex projective space) and $\mathbb{H} P^{n}$ (the $n$-dimensional quaternion projective space). We use as main tool the action of the map on the homology groups of these compact spaces.
\end{abstract}

\section{INTRODUCTION}

Let $f: \mathbb{X} \rightarrow \mathbb{X}$ be a continuous map on a compact space $\mathbb{X}$. A point $x \in \mathbb{X}$ is periodic of period $n$ if $f^{n}(x)=x$ and $f^{k}(x) \neq x$ for $k=1, \ldots, n-1$. We denote by $\operatorname{Per}(f)$ the set of periods of all periodic points of $f$. The aim of the present paper is to provide some information on $\operatorname{Per}(f)$ for some compact spaces. More precisely, we shall present results for the spaces $\mathbb{X} \in \Delta$, where $\Delta$ is the set formed by the spaces: $\mathbb{S}^{n}$ (the $n$-dimensional sphere), $\mathbb{S}^{n} \times \mathbb{S}^{m}$ (the product space of the $n$-dimensional with the $m$-dimensional spheres), $\mathbb{C} P^{n}$ (the $n$-dimensional complex projective space) and $\mathbb{H} P^{n}$ (the $n$-dimensional quaternion projective space).

The statement of our main results are the following ones.

Theorem 1. Let $f$ be a continuous self-map on $\mathbb{S}^{n}$ of degree $D$. Then the following statements hold.

(a) If $n$ is even and $D=-1$, then $\operatorname{Per}(f) \cap\{1,2\} \neq \emptyset$.

(b) If $n$ is odd and $D \neq 1$, then $\operatorname{Per}(f) \cap\{1\} \neq \emptyset$.

Theorem 2. Let $f$ be a continuous self-map on $\mathbb{S}^{n} \times \mathbb{S}^{n}$ of degree $D$, and let $f_{* n}=\left(\begin{array}{ll}a & b \\ c & d\end{array}\right)$ with $a, b, c, d \in \mathbb{Z}$, the action of $f$ on the $n$-th homology group $H_{n}\left(\mathbb{S}^{n} \times \mathbb{S}^{n}, \mathbb{Q}\right) \approx \mathbb{Q} \oplus \mathbb{Q}$. Then the following statements hold.

(a) Assume $n$ is even.

(a.1) If $1+a-d+D \neq 0$, then $\operatorname{Per}(f) \cap\{1\} \neq \emptyset$.

(a.2) If $1+a-d+D=0$ and $1+a^{2}+2 b c+d^{2}+D^{2} \neq 0$, then $\operatorname{Per}(f) \cap\{1,2\} \neq \emptyset$

Key words and phrases. Periods, periodic points, continuous map, Lefschetz fixed point theory, sphere, product of two spheres, complex projective space, quaternion projective space. 2010 Mathematics Subject Classification: 37C25, 37C30. 
(a.3) If $1+a-d+D=1+a^{2}+2 b c+d^{2}+D^{2}=0$ and $1+a^{3}+3 a b c+$ $3 b c d+d^{3}+D^{3} \neq 0$ then $\operatorname{Per}(f) \cap\{1,3\} \neq \emptyset$.

(a.4) If $D \neq-1$, then $\operatorname{Per}(f) \cap\{1,2,3\} \neq \emptyset$.

(a.5) If $D=-1$, then $\operatorname{Per}(f) \cap\{1,2,3,4\} \neq \emptyset$.

(b) Assume $n$ is odd.

(b.1) If $1-a-d+D \neq 0$, then $\operatorname{Per}(f) \cap\{1\} \neq \emptyset$.

(b.2) If $1-a-d+D=0$ and $1-a^{2}-2 b c-d^{2}+D^{2} \neq 0$, then $\operatorname{Per}(f) \cap\{1,2\} \neq \emptyset$.

Theorem 3. Let $f: \mathbb{S}^{n} \times \mathbb{S}^{m} \rightarrow \mathbb{S}^{n} \times \mathbb{S}^{m}$ with $n \neq m$ be a continuous map of degree $D, f_{* n}=(a), f_{* m}=(b)$ with $a, b \in \mathbb{Z}$. Here for $k=n, m, f_{* k}$ denotes the action on the $k$-th homology group $H_{k}\left(\mathbb{S}^{n} \times \mathbb{S}^{m}, \mathbb{Q}\right) \approx \mathbb{Q}$. Then the following statements hold.

(a) Assume that $n$ and $m$ are even.

(a.1) If $(a, b) \neq(-1,-1)$, then $\operatorname{Per}(f) \cap\{1\} \neq \emptyset$.

(a.2) If $(a, b)=(-1,-1)$, then $\operatorname{Per}(f) \cap\{1,2\} \neq \emptyset$.

(b) Assume that $n$ and $m$ are odd. Then $(a, b)=(1,1)$, then $\operatorname{Per}(f) \cap\{1\} \neq$ $\emptyset$.

(c) Assume that $n$ is odd and $m$ is even. Then $(a, b)=(1,-1)$, then $\operatorname{Per}(f) \cap\{1\} \neq \emptyset$.

Theorem 4. Let $f: \mathbb{X} \rightarrow \mathbb{X}$ be a continuous map and let $\mathbb{X}$ be either $\mathbb{C} P^{n}$ or $\mathbb{H} P^{n}$. If $f_{* k}$ denotes the action on the $k$-th homology group $H_{k}(\mathbb{X}, \mathbb{Q}) \approx \mathbb{Q}$, with $k=2 n$ if $\mathbb{X}=\mathbb{C} P^{n}$, and $k=4 n$ if $\mathbb{X}=\mathbb{H} P^{n}$ the actions $f_{* 2 n}=f_{* 4 n}=\left(a^{n}\right)$.

(a) If $n$ is odd and $a=-1$, then $\operatorname{Per}(f) \cap\{1,2\} \neq \emptyset$.

(b) If the assumptions of statement (a) do not hold, then $\operatorname{Per}(f) \cap\{1\} \neq \emptyset$.

These four theorems are proved in the next section.

Similar results on the spaces of $\Delta$ for $C^{1}$ self-maps where stated in [5], for transversal self-maps in [10] and [6] except for the case of the $n$-dimensional sphere which is monographically studied in [8]. Also results of the same kind for continuous-self maps on compact surfaces where obtained in [7].

\section{Proofs of Theorems $1,2,3$ And 4}

Assume that $\mathbb{X} \in \Delta$ with dimension $n$ and let $f: \mathbb{X} \rightarrow \mathbb{X}$ be a continuous map, there exist $n+1$ induced linear maps $f_{* k}: H_{k}(\mathbb{X}, \mathbb{Q}) \rightarrow H_{k}(\mathbb{X}, \mathbb{Q})$ for $k=0,1, \ldots, n$ by $f$. Every linear map $f_{* k}$ is given by an $n_{k} \times n_{k}$ matrix with integer entries, where $n_{k}$ is the dimension of $H_{k}(\mathbb{X}, \mathbb{Q})$.

In this setting is defined the Lefschetz number $L(f)$ as

$$
L(f)=\sum_{k=0}^{n}(-1)^{k} \operatorname{trace}\left(f_{* k}\right) .
$$

The importance of this notion is given by the existence of a result connecting the algebraic topology with the fixed point theory called the Lefschetz Fixed 
Point Theorem which establishes the existence of a fixed point if $L(f) \neq 0$, see for instance [1].

Since our aim is to obtain information on the set of periods of $f$ for continuous self-maps of $\Delta$, it is useful to have information on the whole sequence $\left\{L\left(f^{m}\right)\right\}_{m=0}^{\infty}$ of the Lefschetz numbers of all iterates of $f$. Thus we define the Lefschetz zeta function of $f$ as

$$
\mathcal{Z}_{f}(t)=\exp \left(\sum_{k=1}^{\infty} \frac{L\left(f^{k}\right)}{k} t^{k}\right) .
$$

This function generates the whole sequence of Lefschetz numbers, and it may be independently computed through

$$
\mathcal{Z}_{f}(t)=\prod_{k=0}^{n} \operatorname{det}\left(I_{n_{k}}-t f_{* k}\right)^{(-1)^{k+1}}
$$

where $I_{n_{k}}$ is the $n_{k} \times n_{k}$ identity matrix, and we take $\operatorname{det}\left(I_{n_{k}}-t f_{* k}\right)=1$ if $n_{k}=0$. Note that the expression (2) is a rational function in $t$. So the information on the infinite sequence of integers $\left\{L\left(f^{k}\right)\right\}_{k=0}^{\infty}$ is contained in two polynomials with integer coefficients, for more details see [3].

In short the Lefschetz zeta function is a good tool for studying the existence of periodic points and we shall see here, and also for studying the non existence of such points as was shown in $[4,9]$.

Proof of Theorem 1. For $n \geq 1$ let $f: \mathbb{S}^{n} \rightarrow \mathbb{S}^{n}$ be a continuous map. The homological groups of $\mathbb{S}^{n}$ over $\mathbb{Q}$ and the induced linear maps are of the form

$$
H_{q}\left(\mathbb{S}^{n}, \mathbb{Q}\right)= \begin{cases}\mathbb{Q} & \text { if } q \in\{0, n\} \\ 0 & \text { otherwise }\end{cases}
$$

where $f_{* 0}=(1), f_{* i}=(0)$ for $i=1, \ldots, n-1$ and $f_{* n}=(D)$ where $D$ is the degree of the map $f$, see for more details [2].

From (2) we have that

$$
\mathcal{Z}_{f}(t)=\frac{(1-D t)^{(-1)^{n+1}}}{1-t}
$$

If $n$ is even, then

$$
\sum_{k=1}^{\infty} \frac{L\left(f^{k}\right)}{k} t^{k}=\sum_{k=1}^{\infty} \frac{1+D^{k}}{k} t^{k}
$$

so from (1) we get $L\left(f^{k}\right)=1+D^{k}$. Hence if $D=-1, L\left(f^{k}\right)=0$ if $k$ is odd and $L\left(f^{k}\right)=2$ if $k$ is even. Consequently, by the Lefschetz fixed point theorem we have $\operatorname{Per}(f) \cap\{1,2\} \neq \emptyset$. This proves statement (a).

If $n$ is odd, then

$$
\sum_{k=1}^{\infty} \frac{L\left(f^{k}\right)}{k} t^{k}=\sum_{k=1}^{\infty} \frac{1-D^{k}}{k} t^{k}
$$


so from (1) we get $L\left(f^{k}\right)=1-D^{k}$. Hence, if $D=1$ then $L\left(f^{k}\right)=0$ for $k \geq 1$, if $D=-1$ then $L\left(f^{k}\right)=0$ if $k$ is even and $L\left(f^{k}\right)=2$ if $k$ is odd, if $D \neq \pm 1$ then $L\left(f^{k}\right) \neq 0$ for $k \geq 1$. Consequently, by the Lefschetz fixed point theorem we have $\operatorname{Per}(f) \cap\{1\} \neq \emptyset$. This proves statement (b).

Proof of Theorem 2. Let $f$ be a continuous self-map of $\mathbb{S}^{n} \times \mathbb{S}^{n}$. We know that the induced linear maps are $f_{* 0}=(1), f_{* n}=\left(\begin{array}{ll}a & b \\ c & d\end{array}\right)$ with $a, b, c, d \in \mathbb{Z}$, $f_{* 2 n}=(D)$, where $D$ is the degree of the map $f$ and $f_{* i}=(0)$ for $i \in\{0, \ldots, 2 n\}$, $i \neq 0, n, 2 n$ (see for more details [2]). From (2) the Lefschetz zeta function of $f$ is

$$
\mathcal{Z}_{f}(t)=\frac{p(t)^{(-1)^{n+1}}}{(1-t)(1-D t)}
$$

where $p(t)=1-(a+d) t+(a d-b c) t^{2}$.

If $n$ even from the definition of the Lefschetz zeta function in (1) in (3) we have that

$$
\begin{aligned}
\sum_{k=1}^{\infty} \frac{L\left(f^{k}\right)}{k} t^{k}= & \log \left(\frac{1}{\left(1-(a+d) t+(a d-b c) t^{2}\right)(1-t)(1-D t)}\right) \\
= & (1+a+d+D) t+\frac{1}{2}\left(1+a^{2}+2 b c+d^{2}+D^{2}\right) t^{2} \\
& +\frac{1}{3}\left(1+a^{3}+3 a b c+3 b c d+d^{3}+D^{3}\right) t^{3} \\
& +\frac{1}{4}\left(1+a^{4}+4 a^{2} b c+2 b^{2} c^{2}+4 a b c d+4 b c d^{2}+d^{4}+D^{4}\right) t^{4}+\ldots
\end{aligned}
$$

If $L(f)=1+a+d+D \neq 0$, then $\operatorname{Per}(f) \cap\{1\} \neq \emptyset$, and statement (a.1) is proved. If $L(f)=0$ and $L\left(f^{2}\right)=1+a^{2}+2 b c+d^{2}+D^{2} \neq 0$, then $\operatorname{Per}(f) \cap\{1,2\} \neq \emptyset$, and it follows statement (a.2). If $L(f)=L\left(f^{2}\right)=0$ and $L\left(f^{3}\right)=1+a^{3}+3 a b c+3 b c d+d^{3}+D^{3} \neq 0$, then $\operatorname{Per}(f) \cap\{1,3\} \neq \emptyset$, proving statement (a.3). If $L(f)=L\left(f^{2}\right)=L\left(f^{3}\right)=0$, then $D=-1$ consequently statement (a.4) is proved. If $D=-1$, the system $L(f)=L\left(f^{2}\right)=L\left(f^{3}\right)=$ $L\left(f^{4}\right)=0$ has no solutions in the variables $a, b, c, d$ and statement (a.5) is proved.

If $n$ is odd, from (1) and (3) we have that

$$
\begin{aligned}
\sum_{k=1}^{\infty} \frac{L\left(f^{k}\right)}{k} t^{k}= & \log \left(\frac{1-(a+d) t+(a d-b c) t^{2}}{(1-t)(1-D t)}\right) \\
= & (1-a-d+D) t+\frac{1}{2}\left(1-a^{2}-2 b c-d^{2}+D^{2}\right) t^{2} \\
& +\frac{1}{3}\left(1-a^{3}-3 a b c-3 b c d-d^{3}+D^{3}\right) t^{3} \ldots
\end{aligned}
$$

We note that if $L(f)=1-a-d+D=0$ and $L\left(f^{2}\right)=1-a^{2}-2 b c-d^{2}+D^{2}=0$, then $\mathcal{Z}_{f}(t)=0$ and in this case we do not have information on the periods of the map $f$. 
If $L(f) \neq 0$, then statement (b.1) follows. If $L(f)=0$ and $L\left(f^{2}\right) \neq 0$, then $\operatorname{Per}(f) \cap\{1,2\} \neq \emptyset$ and statement (b.2) is proved. This completes the proof of the theorem.

Proof of Theorem 3. Let $f$ be a continuous self-map of $\mathbb{S}^{n} \times \mathbb{S}^{m}$ with $n \neq m$. It is known that the induced linear maps are $f_{* 0}=(1), f_{* n}=(a), f_{* m}=(b)$ with $a, b \in \mathbb{Z}, f_{* n+m}=(D)$, where $D \in \mathbb{Z}$ is the degree of the map $f$ and $f_{* i}=(0)$ for $i \in\{0, \ldots, n+m\}, i \neq 0, n, m, n+m$ (see for more details [2]).

By Poincaré duality, or again by a direct consideration with the cup-product, we have $\operatorname{deg}(f)=D=a b$, see [11].

From (2) the Lefschetz zeta function of $f$ is of the form

$$
\mathcal{Z}_{f}(t)=\frac{(1-a t)^{(-1)^{n+1}}(1-b t)^{(-1)^{m+1}}(1-a b t)^{(-1)^{n+m+1}}}{1-t} .
$$

Let $f$ be an orientation preserving homeomorphism, $n$ and $m$ even. Therefore the degree $D$ of $f$ is 1 . By (1) and (4) we have that

$$
\begin{aligned}
\sum_{k=1}^{\infty} \frac{L\left(f^{k}\right)}{k} t^{k} & =\log \left(\frac{1}{(1-t)(1-a t)(1-b t)(1-a b t)}\right) \\
& =\sum_{k=1}^{\infty} \frac{1+a^{k}+b^{k}+a^{k} b^{k}}{k} t^{k}
\end{aligned}
$$

Therefore, $L\left(f^{k}\right)=1+a^{k}+b^{k}+a^{k} b^{k}$. If $(a, b) \neq(-1,-1)$, then $L(f) \neq 0$ and this proves statement (a.1). If $(a, b)=(-1,-1)$, then $L\left(f^{2}\right)=2$ and $\operatorname{Per}(f) \cap\{1,3\} \neq \emptyset$ proving statement (a.2).

Assume that $n$ and $m$ even. Therefore, from the definition of the Lefschetz zeta function (1) and (4) we have that

$$
\begin{aligned}
\sum_{k=1}^{\infty} \frac{L\left(f^{k}\right)}{k} t^{k} & =\log \left(\frac{(1-a t)(1-b t)}{(1-t)(1-a b t)}\right) \\
& =\sum_{k=1}^{\infty} \frac{1-a^{k}-b^{k}+a^{k} b^{k}}{k} t^{k}
\end{aligned}
$$

Therefore, $L\left(f^{k}\right)=1-a^{k}-b^{k}+a^{k} b^{k}$. If $(a, b) \neq(1,1)$, then $L(f) \neq 0$ and this shows statement (b). If $(a, b)=(1,1)$, then $L\left(f^{k}\right)=0$ for $k \geq 1$.

Assume that $n$ odd and $m$ even. Therefore, from the definition of the Lefschetz zeta function (1) and (4) we have that

$$
\begin{aligned}
\sum_{k=1}^{\infty} \frac{L\left(f^{k}\right)}{k} t^{k} & =\log \left(\frac{(1-a t)(1-a b t)}{(1-t)(1-b t)}\right) \\
& =\sum_{k=1}^{\infty} \frac{1-a^{k}+b^{k}-a^{k} b^{k}}{k} t^{k}
\end{aligned}
$$

Therefore, $L\left(f^{k}\right)=1-a^{k}+b^{k}-a^{k} b^{k}$. If $(a, b) \neq(1,-1)$, then $L(f) \neq 0$ and this shows statement (c). If $(a, b)=(1,-1)$, then $L\left(f^{k}\right)=0$ for $k \geq 1$, ending the proof of the theorem. 
Proof of Theorem 4. Let $f$ be a continuous self-map of $\mathbb{C} P^{n}$ with $n \geq 1$. We know that the induced linear maps are $f_{* q}=\left(a^{\frac{q}{2}}\right)$ for $q \in\{0,2,4, \ldots, 2 n\}$ with $a \in \mathbb{Z}$, and $f_{* q}=(0)$ otherwise (see for more details [12, Corollary 5.28]).

From (2) the Lefschetz zeta function of $f$ has the form

$$
\mathcal{Z}_{f}(t)=\left(\prod_{q}\left(1-a^{q / 2} t\right)\right)^{-1},
$$

where $q$ runs over $\{0,2,4, \ldots, 2 n\}$.

Let $f$ be a continuous self-map of $\mathbb{H} P^{n}$ with $n \geq 1$. We know that the induced linear maps are $f_{* q}=\left(a^{\frac{q}{4}}\right)$ for $q \in\{0,4,8, \ldots, 4 n\}$ with $a \in \mathbb{Z}$, and $f_{* q}=(0)$ otherwise (see for more details [12, Corollary 5.33]).

From (2) the Lefschetz zeta function of $f$ has the form

$$
\mathcal{Z}_{f}(t)=\left(\prod_{q}\left(1-a^{q / 4} t\right)\right)^{-1}
$$

where $q$ runs over $\{0,4,8, \ldots, 4 n\}$.

By (1), (5) and (6) we have that

$$
\sum_{k=1}^{\infty} \frac{L\left(f^{k}\right)}{k} t^{k}=\sum_{k=1}^{\infty} \frac{a^{k(n+1)-1}}{a^{k}-1} t^{k}
$$

Therefore $L\left(f^{k}\right)=\frac{a^{k(n+1)-1}}{a^{k}-1}$. Hence it is easy to check first that $L(f)=0$ if and only if $n$ is odd and $a=-1$, and second that $L\left(f^{2}\right)=1+a^{2}+\ldots+a^{2 n} \neq 0$. From these two facts the statements (a) and (b) follow.

\section{ACKNOWLEDGEMENTS}

The first author of this work was partially supported by MINECO grant number MTM2014-51891-P and Fundación Séneca de la Región de Murcia grant number $19219 / \mathrm{PI} / 14$.. The second author is partially supported by a MINECO grant MTM2013-40998-P, an AGAUR grant number 2014SGR-568, and the grants FP7-PEOPLE-2012-IRSES 318999 and 316338.

\section{REFERENCES}

[1] R.F. Brown, The Lefschetz Fixed Point Theorem, Scott, Foresman and Company, Glenview, IL, 1971.

[2] C.T. Dodson And P.E. PARKer, A user's guide to algebraic topology, Kluwer Acad. Publ., 1996.

[3] J. Franks, Homology and Dynamical Systems, CBMS Regional Conf. Series, vol. 49, Amer. Math. Soc., Providence R.I., 1982.

[4] J.L.G. Guirao And J. Llibre, On the Lefschetz periodic point free continuous selfmaps on connected compact manifolds, Topology and its Applications 158 (2011), 21652169

[5] J.L.G. Guirao and J. Llibre, $\mathcal{C}^{1}$ self-maps on $\mathbb{S}^{n}, \mathbb{S}^{n} \times \mathbb{S}^{m}, \mathbb{C} P^{n}$ and $\mathbb{H} P^{n}$, Taiwanese Journal of Mathematics 16 (2012), 323-334 
[6] J.L.G. Guirao And J. Llibre, Periodic structure of transversal maps on $\mathbb{C} P^{n}$ and $\mathbb{H} P^{n}, \mathbb{S}^{p} \times \mathbb{S}^{q}$, Qualitative Theory of Dynamical Systems 12(2) (2013), 417-425

[7] J.L.G. Guirao and J. Llibre, Periods of continuous maps on closed surfaces, Rocky Mountain J. Math., To appear.

[8] J. Llibre, A note of the set of periods of transversal homological sphere self-maps, Journal of Difference Equations and Applications. 9, (2003), 417-422

[9] J. Llibre, Periodic point free continuous self-maps on graphs and surfaces, Topology and its Applications 159 (2012), 2228-2231

[10] J. Llibre, J. Paraños And J.A. Rodriguez, Periods for transversal maps on compact manifold with a given homology, Houston J. of Math. 4, (1998), 397-407.

[11] A. Hatcher, Algebraic topology, Cambridge University Press, 2002.

[12] J.W. VICKs, Homology theory. An introduction to algebraic topology, Springer-Verlag, New York, 1994. Academic Press, New York, 1973.

${ }^{1}$ Departamento de Matemática Aplicada y Estadística. Universidad Politécnica de Cartagena, Hospital de Marina, 30203-Cartagena, Región de Murcia, SPAIN.

E-mail address: juan.garcia@upct.es

${ }^{2}$ Departament de Matemàtiques. Universitat Autònoma de Barcelona, Bellaterra, 08193-Barcelona, Catalonia, Spain

E-mail address: jllibre@mat.uab.cat 